\title{
Echocardiographic findings in pulmonary embolism: An important guide for the management of the patient
}

\author{
Ronny Cohen $^{1,2}$, Pablo Loarte ${ }^{2}$, Victor Navarro ${ }^{1,2}$, Brooks Mirrer ${ }^{1,2}$ \\ ${ }^{1}$ NYU School of Medicine, New York, USA \\ ${ }^{2}$ Woodhull Medical Center, New York, USA \\ Email: ronny.cohen@nychhc.org
}

Received 23 December 2011; revised 6 March 2012; accepted 4 June 2012

\begin{abstract}
Echocardiography can be used as an easy, inexpensive, devoid of complications and, for the most part, universally available tool for the risk stratification of patient with acute pulmonary embolism. Because of its low sensibility, an echocardiogram is best indicated in a patient in whom hypotension and or shock are suspected to be due to pulmonary embolism. Pertinent findings in the echocardiogram can justify the use of fibrinolytic therapy. Most common echocardiographic findings in acute pulmonary embolism are: dilatation of the right ventricle, right ventricular dysfunction in some cases with preservation of the motility of the apex, dilatation of the inferior vena cava with lack of collapse during inspiration flattening of the interventricular septum suggesting right ventricular pressure overload and pulmonary hypertension based on the jet of tricuspid regurgitation if available.
\end{abstract}

Keywords: Pulmonary Embolism; Echocardiography; Venous Thromboembolism; Deep Vein Thrombosis

\section{INTRODUCTION}

Pulmonary embolism (PE) is a serious medical condition, potentially fatal, caused by partial or total obstruction of the pulmonary arteries and or its branches usually by blood clots but also air, tissue and fat. The most common cause is secondary embolization from thrombosis of veins usually originating from lower extremities or pelvis. Risk factors for thrombosis include endothelial injury, hypercoagulability of blood and stasis [1]. PE is an important cause of patient morbidity and mortality, with estimated 200,000 patient deaths per year in the US. The mortality reaches $30 \%$ for untreated PE; however with treatment the mortality rate decreases to $2.5 \%-10 \%$ [2]. It is estimated that more than half of all $\mathrm{PE}$ are probably undiagnosed and frequently not discovered until autopsy [2].
Symptoms of PE include chest pain, hemoptysis, and dyspnea. Because the clinical presentation tends to be very nonspecific, diagnostic testing plays a crucial role in the diagnosis. Evaluation should include history taking, chest $\mathrm{x}$-ray, electrocardiogram, D-dimer assay (high sensitivity but poor specificity), computed tomography (sensitivity $53 \%-100 \%$, specificity of $83 \%-100 \%$ ), ventilation-perfusion scan (high sensitivity but poor specificity). Although pulmonary angiography is the "gold" standard of reference for confirming or refuting a diagnosis of $\mathrm{PE}$ [3], spiral CT with contrast is the most widely used modality for diagnosis of pulmonary embolism.

ECG findings are usually nonspecific. In fact, $33 \%$ of patients with pulmonary embolism have a normal ECG. The most common abnormal findings are sinus tachycardia and nonspecific ST-segment and T-wave changes. Changes that strongly suggest pulmonary embolism indicate strain on the right side of the heart; manifesting as $\mathrm{T}$-wave inversion in precordial leads $\mathrm{V}_{1}$ through $\mathrm{V}_{4}$, transient right bundle branch block, new right or left deviation of the QRS axis, sudden onset of atrial fibrillation or other atrial arrhythmia, and right atrial enlargement may be seen [4].

The $\mathrm{S}_{1} \mathrm{Q}_{3} \mathrm{~T}_{3}$ pattern (prominence of $\mathrm{S}$ wave in lead $\mathrm{I}, \mathrm{Q}$ wave in lead III, and T-wave inversion in lead III) also suggests pulmonary embolism [4].

Echocardiography may play a significant role in making therapeutic decisions in patients with pulmonary embolism. This paper discusses the utility of using echocardiogram in diagnosing and guiding treatment in patients with pulmonary embolism.

Echocardiography is most appropriately recommended for patients with hypotension and or shock and suspected pulmonary embolism.

\section{ECHOCARDIOGRAPHIC FINDING IN ACUTE PULMONARY EMBOLISM INCLUDE}

Right ventricular enlargement, right ventricular dysfunction, in some cases with preservation of the apical con- 
tractility (McConnell's sign). When the pulmonary embolism is large, more than $90 \%$ of patients have right ventricular hypokinesis [5].

Signs of pulmonary hypertension: flattening of the interventricular septum during systole, tricuspid regurgitant flow velocity higher than $2.7 \mathrm{~m} / \mathrm{sec}$.

In patients with acute PE there is marked prolongation of the isovolumic contraction time and isovolumic relaxation time over the tricuspid annulus. During chronic pulmonary hypertension the isovolumetric contraction and relaxation time of the $\mathrm{LV}$ is also increased, hence both RVMPI and LVMPI are increased. During acute pulmonary hypertension, because the LV has had no time to adapt, the LVMPI is not increased so there is a discrepant increased of the RVMPI over the LVMPI helping differentiate chronic pulmonary hypertension from acute pulmonary hypertension of $\mathrm{PE}[6,7]$.

Other signs: Short acceleration time of the pulmonary flow velocity in the presence of a tricuspid regurgitation gradient of less than $60 \mathrm{mmHg}$. RV to LV diameter ratio $>1[8]$.

Thrombus seen within the right sided chambers [8].

However, echocardiography shows indirect evidence of pulmonary embolism in about $80 \%$ of patients with massive embolism (i.e., $\geq 60 \%$ perfusion defects) [9].

Echocardiography may directly visualize embolized thrombi (right heart chambers or central pulmonary arteries) or show right heart hemodynamic changes that indirectly suggest pulmonary embolism. For this a transesophageal echocardiogram holds a higher promise as a diagnostic tool $[7,9]$.

\section{ECHOCARDIOGRAM IN ESTIMATION OF PROGNOSIS IN PULMONARY EMBOLISM}

The presence of right ventricular hypokinesis, among other factors, is a sign of adverse prognosis for the patient [10].

The finding of a patent foramen ovale in conjunction with pulmonary embolism indicates a relatively poor short-term prognosis and may encourage the use of more aggressive therapy for PE [10].

A study done by Stein et al. showed that the in hospital prognosis of patients with right ventricular enlargement following a pulmonary embolism is good in the absence of shock, a life threatening arrhythmia or ventilatory support [11].

\section{USE OF ECHOCARDIOGRAPHY AS A TOOL FOR DIFFERENTIAL DIAGNOSIS}

Echocardiography can help differentiate pulmonary embolism from other causes of hypotension: pneumothorax, pericardial effusion, myocardial infarction and sepsis.

In conjunction with clinical assessment and the results of other noninvasive tests, echocardiography may enable $\mathrm{PE}$ to be diagnosed, or anticoagulants to be withheld in severely ill patients, at least until it becomes feasible to perform additional testing.

In addition, indirect parameters such as unexplained right ventricular dilatation/dysfunction and marked tricuspid regurgitation, which can be detected similarly by TTE and TEE, have a sensitivity of about $50 \%$ and a specificity of about $90 \%$ for pulmonary embolism.

\section{TTE VS. TEE}

With the transesophageal echocardiography it is possible to see the right-sided cardiac structures, the main pulmonary artery, a significant portion of the right pulmonary artery, and a portion of the left pulmonary artery. [12].

TTE visualizes intracardiac thrombi (usually right atrium) in about $5 \%$ of patients with acute pulmonary embolism and generally does not detect emboli in the pulmonary arteries. Central emboli can be seen by TEE in about $70 \%$ of the patients who have pulmonary embolism and right ventricular dysfunction. TEE can visualize thrombi in the central pulmonary arteries (main, right, proximal portion of left) with high specificity $(>90 \%)$, but its sensitivity has not been evaluated in unselected patients with pulmonary embolism (perhaps about 30\%) [13-16].

Because of the limited specificity with the TTE, the invasiveness of the TEE and the low sensitivity with both approaches, echocardiography is not suitable as a routine diagnostic test for PE. Outside the ICU, as a stand-alone imaging technique for the diagnosis of $\mathrm{PE}$, echocardiography should not be used. It is rather insensitive (reported range, $30 \%$ to $50 \%$ ). Findings on echocardiography, even in patients with shock suspected to be due to a PE, are nonspecific. Although certain findings, such as free-floating thrombus or clot transiting through the right heart, are diagnostic for PE, these are rare findings. In the ICU, however, echocardiography is attractive since it is noninvasive and does not require the transport of an unstable patient. Moreover, $80 \%$ of patients with shock and PE show evidence of right-heart dysfunction on echocardiography. However, in the patient with unexplained shock, the differential diagnosis is broad and includes a number of processes that can cause both right ventricular dilation and dysfunction. Even if echocardiography is nondiagnostic for $\mathrm{PE}$, it may still provide important information by helping to exclude other causes of shock, such as acute left ventricular dysfunction, tamponade, acute valvular disease, and aortic dissection, as noted above $[14,15]$. 


\section{CURRENT THERAPIES FOR PE}

Accurate diagnosis followed by effective therapy with anticoagulants decreases mortality. Conventional therapy for acute $\mathrm{PE}$ is the administration of intravenous heparin or Low Molecular Weight Heparin (LMWH). Heparin prevents clot formation and extension. Because the risk of death from pulmonary embolism is greatest in the first few hours of development of a clot and because diagnostic test results often are not available for 8 to 12 hours, heparin should be given to patients with a moderate to high clinical probability of pulmonary embolism or deep vein thrombosis until all diagnostic results are available. LMWH is preferred to unfractionated heparin. LMWH can be given subcutaneously once or twice a day, and laboratory monitoring may not be necessary. Long-term anticoagulation is continued after discharge, usually with warfarin

Thrombolytic therapy should be considered for patients with deep vein thrombosis involving the iliofemoral system. Thrombolytic therapy accelerates the resolution rate of pulmonary occlusion and improves right ventricular function. These effects are seen within 24 hours, as opposed to two weeks with heparin or LMWH; therefore thrombolytic agents are used for patients with massive PE and hemodynamic instability or with echocardiographic findings of right ventricular failure. It is also useful for patients with massive pulmonary embolism who have significant pulmonary hypertension, obstruction of multiple segments of the pulmonary circulation, right ventricular dysfunction, or systemic hypotension [17].

Clot lysis may reduce the incidence of recurrent thrombi and postphlebitic syndrome and returns pulmonary arterial pressure to normal faster than heparin Thrombolytic therapy also relieves strain on the right side of the heart faster than heparin does. However, thrombolytic therapy does not improve survival. Risks include hemorrhage, including an approximate $1 \%$ to $2 \%$ risk of intracranial bleeding. The hemodynamic response and the rate of bleeding are similar across age groups. Contraindications to thrombolytic therapy include eye or central nervous system surgery within the preceding 2 weeks, intracranial neoplasms or vascular abnormalities, stroke within the preceding 2 months, active bleeding, severe hypertension, and allergy to thrombolytic agents. Patient's age should not be a consideration when deciding on whether to use thrombolytics [16].

Interruption of the inferior vena cava, usually with a Greenfield filter, may be required in patients who have a contraindication to anticoagulation, have recurrent emboli while on anticoagulation, have pulmonary emboli from septic thrombophlebitis or those with massive embolization from a clot in the legs. Endarterectomy may be helpful in patients who have chronic pulmonary hy- pertension due to a clot occluding the main pulmonary artery or a lobar pulmonary artery [16].

\section{PREVENTION OF PE}

Prophylaxis decreases the incidence of fatal pulmonary emboli by two thirds in hospitalized patients at risk of developing venous clots. LMWH (e.g., enoxaparin 40 $\mathrm{mg} \mathrm{sc}$ once daily) is as effective and safe as prophylaxis with subcutaneous heparin (5000 IU sc bid or tid) and may reduce drug-induced adverse effects. Postoperative prophylaxis with LMWH (e.g., enoxaparin $30 \mathrm{mg}$ sc q 12 $\mathrm{h}$ for up to 14 days) also dramatically reduces the incidence of venous thrombosis after knee or hip replacement. For total hip replacement, some investigators find that 4 to 6 weeks of LMWH postoperatively may be more effective [16].

Pulmonary embolism is believed to recur in 5 to $10 \%$ of patients despite ongoing heparin therapy. The likelihood of recurrent emboli is greatest in patients who have massive pulmonary embolization or for whom anticoagulant therapy is inadequate. If recurrence develops in the first few days of heparin or thrombolytic therapy, this treatment is usually continued. Recurrent pulmonary embolism leading to chronic pulmonary hypertension and cor pulmonale is uncommon [15].

\section{CONCLUSION}

Echocardiography, included in this term bedside transthoracic and transesophageal, is a very helpful tool for the clinician that faces acute ill patients in whom the diagnosis of pulmonary embolism is in doubt. By demonstrating sings of right ventricular strain and pulmonary hypertension, echocardiogram is able to help in the treatment of these patients. Echocardiography is also able to determine which patient is at highest risk of an adverse outcome by means of evaluating the right ventricular function.

\section{REFERENCES}

[1] Senior, R.M. (1996) Pulmonary embolism. In: Bennett, J.C., et al., Eds., Cecil Textbook of Medicine, 20th Edition, Saunders, Philadelphia, 422-429.

[2] Horlander, K.T., Mannino, D.M. and Leeper, K.V. (2003) Pulmonary embolism mortality in the United States, 1979-1998: An analysis using multiple-cause mortality data. Archives of Internal Medicine, 163, 1711-1717. doi:10.1001/archinte.163.14.1711

[3] Kelley, M.A., et al. (1991) Diagnosing pulmonary embolism: New facts and strategies. Annals of Internal Medicine, 114, 300-306.

[4] Ginghina, C., Caloianu, G.A., Serban, M. and Dragomir, D. (2010) Right ventricular myocardial infarction and pulmonary embolism differential diagnosis - A challenge 
for the clinician. Journal of Medicine and Life, 3, 242-253.

[5] Mookadam, F., Jiamsripong, P., Goel, R., Warsame, T.A., Emani, U.R. and Khandheria, B.K. (2010) Critical appraisal on the utility of echocardiography in the management of acute pulmonary embolism. Cardiology in Review, 18, 29-37. doi:10.1097/CRD.0b013e3181c09443

[6] Hsiao, S.H., Lee, C.Y., Chang, S.M., Yang, S.H., Lin, S.K. and Huang, W.C. (2006) Pulmonary embolism and right heart function: Insights from myocardial Doppler tissue imaging. Journal of the American Society of Echocardiography, 19, 822-888. doi:10.1016/j.echo.2006.01.011

[7] Serafini, O., Greco, F., Misuraca, G., Chiatto, M., Buffon, A. (2005) Echocardiography in the diagnostic and prognostic evaluation of thromboembolic pulmonary hypertension. Archives for Chest Disease, 64, 116-123.

[8] Stawicki, S.P., Seamon, M.J., Meredith, D.M., Chovanes, J., Paszczuk, A., Kim, P.K. and Gracias, V.H. (2008) Transthoracic echocardiography for suspected pulmonary embolism in the intensive care unit: Unjustly underused or rightfully ignored? Journal of Clinical Ultrasound, 36, 291-302. doi:10.1002/jcu.20461

[9] Kearon, C. (2003) Diagnosis of pulmonary embolism. Canadian Medical Association Journal, 168, 183-194.

[10] Goldhaber, S.Z., Visani, L. and De Rosa, M. (1999) Acute pulmonary embolism: clinical outcomes in the International Cooperative Pulmonary Embolism Registry (ICOPER). Lancet, 353, 1386-1389. doi:10.1016/S0140-6736(98)07534-5

[11] Stein, P.D., et al. (2008) Enlarged right ventricle without

\section{ABBREVIATION}

PE: pulmonary embolism.

$\mathrm{RV}$ : right ventricle.

LV: left ventricle.

RVMPI: right ventricular myocardial performance index. shock in acute pulmonary embolism: Prognosis. American Journal of Medicine, 121, 134-142.

doi:10.1016/j.amjmed.2007.06.032

[12] Goldhaber, S.Z. (2002) Echocardiography in the management of pulmonary embolism. Annals of Internal Medicine, 136, 691-700.

[13] O’Neill, J.O., Iqbal, R. and McGarry, K. (2002) Thrombus in transit-The role of echocardiography in the diagnosis of massive pulmonary embolism and a review of the literature. Acta Cardiologica, 57, 291-294.

[14] Leibowitz, D. (2001) Role of echocardiography in the diagnosis and treatment of acute pulmonary thromboembolism. Journal of the American Society of Echocardiography, 14, 921-926. doi:10.1067/mje.2001.114390

[15] Torbicki, A., Tramarin, R. and Morpurgo, M. (1992) Role of echo Doppler in the diagnosis of pulmonary embolism. Clinical Cardiology, 15, 805-810. doi:10.1002/clc.4960151104

[16] Bova, C., Greco, F., Misuraca, G., et al. (2003) Diagnostic utility of echocardiography in patients with suspected pulmonary embolism. American Journal of Emergency Medicine, 21, 180-183. doi:10.1016/S0735-6757(02)42257-7

[17] Stein, P.D., Hull, R.D. and Raskob, G. (1994) Risks for major bleeding from thrombolytic therapy in patients with acute pulmonary embolism: Consideration of noninvasive management. Annals of Internal Medicine, 121 313-317.

LVMPI: left ventricular myocardial performance index. TEE: tranesophageal echocardiography.

TTE: transthoracic echocardiography.

LMWH: low molecular weight herparin.

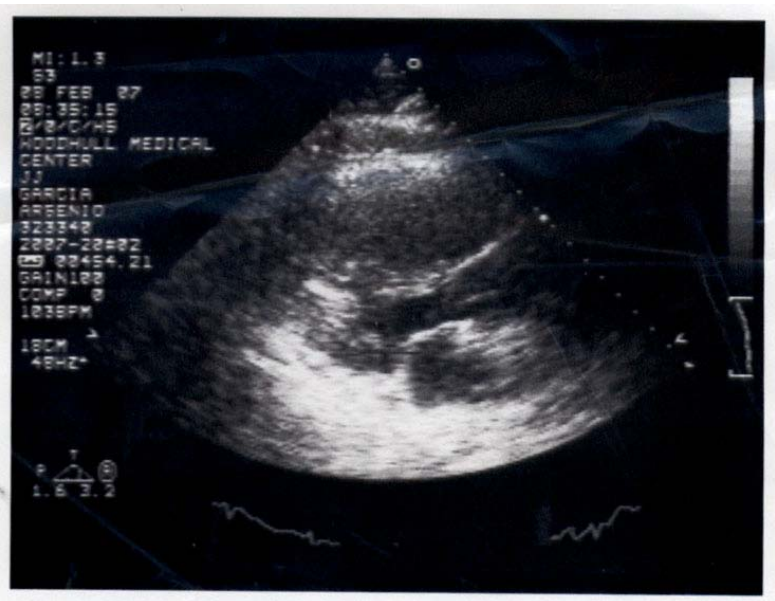

